\title{
Nosocomial Salmonellosis
}

Salmonella infections are an important source of morbidity in the United States, accounting for over 500 outbreaks each year and approximately two-thirds of foodborne outbreaks reported to the Centers for Disease Control. Up to $35 \%$ of reported Salmonella epidemics are in hospitals or chronic care facilities. ${ }^{1,2}$ Nosocomial outbreaks due to Salmonella have been second in frequency only to those caused by staphylococci. ${ }^{3}$ A recent review of data from the National Nosocomial Infection Study (1980-1984) suggests that Clostridium difficile is the most common recognized cause of nosocomial gastroenteritis (J.M. Hughs, Centers for Disease Control, personal communication). Epidemic salmonellosis is well recognized in acute care hospitals, particularly pediatric wards and newborn nurseries, as well as chronic care facilities, such as psychiatric institutions and homes for the elderly.

The importance and complexity of Salmonella as a cause of nosocomial infection is emphasized by the impressive story of an outbreak of Salmonella drypool occurring over a 5-year period reported by Linnemann et al in this issue of Infection Control. This outbreak illustrates classical features of both common source and person-to-person spread. The initial foodborne outbreak was followed by an extremely prolonged and difficult persistence of the infection among food handlers, medical personnel and patientsall at substantial personal and financial cost. This outbreak demonstrates the difficulties encountered in containing an epidemic of salmonellosis. It also raises important questions about the infectious dose, mode of transmission in the hospital, duration of carriage, method of diagnosis and questions about treatment of carriers of nontyphoidal Salmonella.

The widely accepted dictum that a large inoculum of Salmonella is required to cause illness in humans is based largely on the results of studies in volunteers. A recent review by Blaser et al casts significant doubt on this dictum. ${ }^{4}$ In six of 11 evaluable "natural" outbreaks, the actual dose of Salmonella ingested in the outbreak was calculated to be less than $10^{3}$ organisms. In contrast, in eight of nine studies where Salmonella infection was experimentally induced in humans, the lowest dose evaluated in two

From the Division of Geographic Medicine. Uninersity of Virginia Medical Cienter, Charlottessille, Virginia.

Address reprint requests to Richard L. Guerrant. MD. Untersity of Virginia, Dizision of Geographic Medicine, Room 3915. Uniremsity of Virginia Medical Center, Charlontessille. VA 22908. studies was $10^{3}$ organisms but the lowest dose causing illness, in any series, was $10^{5}$ organisms. However, as few as 25 organisms of Salmonella sofia and Salmonella bovis-morbicians into the left nasal antrum of a volunteer resulted in gastrointestinal illness within 37 hours suggesting that the route and method of administration may alter the infectious dose. A relationship between attack rates, incubation periods and the calculated doses was documented in these outbreaks, with the higher calculated doses causing higher attack rates and shorter incubation periods.

An analysis of factors that may influence the course of natural infections reveals several important observations. First, Salmonella of different serotypes vary in their virulence for humans. Three biochemically distinct species of Salmonella exist, Salmonella typhi, Salmonella choleraesuis and Salmonella enteritidis. Salmonella typhi and Salmonella choleraesuis consist of one serotype each. However, the Salmonella enteritidis group includes over 2,000 serotypes, the specific serotype being determined by detailed antigenic analysis which includes the somatic $(\mathrm{O})$ and flagellar $(\mathrm{H})$ antigens. Of these serotypes, Salmonella typhimurium remains the most common accounting for a third of all isolates reported to the Centers for Disease Control in 1983. Differences in virulence among serotypes of Salmonella are best illustrated by the fact that bacteremia, with or without gastrointestinal manifestations, is relatively more common with Salmonella choleraesuis paratyphi $\mathrm{A}$ and dublin. ${ }^{5}$

A second important determinant of the course of natural Salmonella infections is the whole range of host factors, a point not discussed in Linnemann's report that might have relevance, particularly to the hospitalized patients. In a recent study of Salmonella dublin infections, for example, persons infected with Salmonella dublin were more likely to have chronic noninfectious diseases and to have ingested antacids than uninfected controls. ${ }^{5}$ Several host factors have been identified as important in Salmonella infections. First, the age of the host is an important determinant of susceptibility to infection with age-specific attack rates being higher for children less than 1 year of age than for any other group. Not only are infections more common in this age group, but the consequences of infection with Salmonella such as bacteremia and meningitis are particularly severe in children. In addition, persons over the age of 60 are at greater risk for the development of bacteremia. Identified defects in host defenses such as 
achlorhydria (more common in the young and elderly), gastrectomy, malignancy, liver disease, hemoglobinopathy such as sickle cell disease and hemolytic conditions including bartonellosis are important risk factors for Salmonella infections. Finally, prior ingestion of antibiotics reduces the dose of Salmonella necessary to establish infection with both drug-sensitive and drug-resistant strains.

The mechanism of spread in the epidemic reported by Linnemann et al suggests a combination of person-toperson and foodborne spread. It has been stated in the literature that nosocomial outbreaks resulting from asymptomatic Salmonella carriers have not been widely recognized.6,7 However, the role of an asymptomatic chronic excretor, as appropriately suspected by Linnemann et al, should certainly be considered in the setting of an outbreak. In the reported outbreak, it is interesting to note that of the 24 kitchen workers who had positive cultures for Salmonella drypool none were symptomatic. In contrast, employees and patients who acquired the infection presumably by eating contaminated food prepared in the hospital kitchen were symptomatic, suggesting inappropriate food handling that resulted in multiplication of organisms in the food prior to its ingestion. These observations support the concept that a few organisms may be required to establish a carrier state, but that larger numbers of organisms may be required to establish symptomatic infections, in normal hosts.

This paper reopens the controversy over when and how to culture patients with Salmonella infections. The median duration of excretion of nontyphoidal Salmonella is approximately 5 weeks after infection, with excretion being more prolonged in children less than 5 years of age, persons with symptomatic infections and persons infected with serotypes other than Salmonella typhimurium. ${ }^{6}$ Therefore convalescent excretion is quite common but persistent excretion of nontyphoidal Salmonella beyond 1 year occurs in less than $1 \%$ of subjects. Historically, stool specimens have been preferred to rectal swabs for the isolation of Salmonella. However, the use of an enrichment broth and incubation for 72 hours may greatly enhance the positive yield. ${ }^{8}$ The use of an enrichment broth (albeit for only 8 hours) in addition to the delays inherent in culturing stool specimens brought from home may account for the equivalent sensitivity of rectal swabs and stool cultures in the isolation of Salmonella in this outbreak. As is well illustrated by this report, intermittent excretion of detectable Salmonella is common, both in the presence or absence of antimicrobial therapy. The criteria used for definition of a negative in this current outbreak, that is three negative cultures within the space of 2 weeks, may not exclude all persistent asymptomatic carriage. Earlier studies have shown that $8 \%$ to $17 \%$ of infected patients may have positive stool cultures again after four to nine consecutive negative examinations. ${ }^{6}$ Furthermore, the method of collection of the stool for culture may significantly influence the detection of carriage. Up to seven consecutive daily rectal swabs have been required to detect $95 \%$ of known long-term carriers. The sensitivity of stool specimens in detecting long-term carriers has been shown to be significantly greater than rectal swabs. This is probably because rectal swabs are insensitive in detecting fewer than $10^{3}$ Salmonella organisms per gram of feces. 6.9

The sources of Salmonella in nosocomial outbreaks have been related to a number of factors including the use of medications or diagnostics, blood products, banked human milk, use of raw eggs or yeast in tube feeding and improper disinfection of devices between patient use. ${ }^{7}$ Recent epidemiological investigations have related approximately one-half of Salmonella outbreaks (which in some instances have been associated with secondary nosocomial spread) to food-producing animals. ${ }^{2}$

An important question regarding the use of antibiotics in control of an outbreak of Salmonella infection is raised by Linnemann and his associates. First, previous data suggest that antibiotic therapy fails to eradicate fecal Salmonella carriage but that cholecystectomy combined with antibiotic therapy may have some utility. ${ }^{10,11}$ This is obviously an extreme recourse in otherwise healthy individuals. However, definite prospective trials of the treatment of asymptomatic carriers of nontyphoidal Salmonella (in contrast to Salmonella typhi) are not available. Therefore, it is unclear whether or not high doses of ampicillin such as 8 to $12 \mathrm{~g}$ daily would be successful in eradicating chronic non-typhoidal Salmonella infections. Second, a number of clinical studies have shown that antibiotic treatment of acute gastroenteritis is associated with prolonged excretion. ${ }^{11,12}$ In a prospective study of the role of neomycin in treatment of acute nontyphoidal Salmonella intestinal infections, $36 \%$ of the neomycin group were still culture-positive compared to $25 \%$ of those given placebo 6 weeks after therapy. ${ }^{13}$ Although we lack additional prospective trials evaluating this question, experimental data in animals are supportive of the hypothesis that antibiotics decrease the infectious dose of Salmonella and may prolong the carrier stage by inhibition of normal components of the fecal flora. " 1 Furthermore, in several outbreaks the ingestion of penicillin drugs in the month prior to the development of salmonellosis appeared to be a risk factor for disease as well as antibiotic resistance. ${ }^{14,15}$ It is of interest that the authors chose to use trimethoprim-sulfamethoxazole in the treatment of their carriers with Salmonella. In the limited data available on the use of this agent for this indication, $54 \%$ to $80 \%$ of individuals cleared the infection. ${ }^{16,17}$ The use of trimethoprim-sulfamethoxazole was interpreted as being instrumental in ending the epidemic reported here and did not seem to result in significant problems with prolonged fecal carriage. However, two of $50(4 \%)$ of individuals treated did persistently excrete Salmonella drypool, one of whom required therapy for 8 weeks to eradicate the infection. Although not reported, it would have been interesting to know the type of specimen used to culture this individual subsequently and how many negative stool cultures had been obtained to verify that she is, in fact, cured. The high incidence of adverse reactions, almost a third of the hospital employees treated in this particular study, is of concern when one considers using trimethoprim-sulfamethoxazole in similar situations in the future. This is much higher than the $8 \%$ or less reaction rate reported overall with use of this medication. ${ }^{18}$ The reasons for this are unclear but further substantiate that 
indiscriminate use of antibiotics is not warranted in Salmonella infections. It is important not to generalize from the results of this study to similar, but less severe, situations. In general, our feeling would be that antibiotics are not indicated in the treatment of carriers of nontyphoidal Salmonella and in addition, antibiotics have a limited, if any, role in the control of nosocomial salmonellosis. The most important factors in control of Salmonella infections are proper food handling, including thorough cooking, prompt refrigeration, proper disinfection and sterilization of equipment and an emphasis on handwashing practices as well as adequate isolation procedures in institutions. The fact that only one sink was available in this kitchen for 52 workers suggests that inadequate facilities and emphasis on hygienic practices may have contributed to the persistence of this epidemic.

Lastly, this article and other reported data emphasize the tremendous personal and economic impact of sporadic Salmonella outbreaks and of nosocomial infections. The cost of 1,520 stool cultures, the need to import food from an outside source while the hospital kitchen was closed and the loss of productive time by many workers in the kitchen underscore the tremendous economic losses incurred in such a situation. Previous analysis of the economic cost in an outbreak of salmonellosis has indicated that the cost to an individual who did not seek medical care averages about $\$ 125$. Patients who are hospitalized incurred a mean cost of about $\$ 1,750 .{ }^{18}$ Highest costs have been related to illness in infants and in the elderly which corresponds with more frequent and longer hospitalizations. However, lost income and productivity has accounted for up to $64 \%$ of the total cost of an outbreak. ${ }^{19,20}$

In summary, this impressive outbreak reported by Linnemann and associates raises several important questions about infectious doses, routes of spread, host susceptibility, detection of carriers, duration of carriage, cost of evaluation and means of controlling a prolonged, complex hospital epidemic. Without question, the mortality of Salmonella infections in institutionalized patients is substantially higher than case fatality rates in the community. ${ }^{1}$ However, the role of antibiotics in the treatment of carriers, even in the nosocomial setting where control of spread is important to the prevention of infections in hospitalized patients, remains unclear. Although this report provides a preliminary basis for the use of antibiotics in control of difficult epidemics, we would encourage the use of more traditional control measures. Their consideration of treating all workers in a kitchen must be weighed against potential risks and side effects seen with treating large numbers of asymptomatic people. Far from providing all of the answers to the questions raised, Linnemann's paper illustrates several important areas where more information is needed to most efficiently evaluate and control Salmonella infections.

\section{REFERENCES}

1. Baine WB, Gangarosa EJ, Bennett JV, et al: Institutional salmonellosis. J Infect Dis 1973; 128:357-360.

2. Holmberg SD, Wells JG, Cohen ML: Animal-to-man transmission of antimicrobial-resistant Salmonella: Investigations of US outbreaks, 1971-1983. Science 1984; 225:833-835.

3. Stamm WE, Weinstein RA, Dixon RE: Comparison of endemic and epidemic nosocomial infections. Am J Med 1981; 70:393-397.

4. Blaser MJ, Newman LS: A review of human salmonellosis: I. Infective Dose. Rev Infect Dis 1982; 4:1096-6.

5. Taylor DN, Bied JM, Munro JS, et al: Salmonella dublin infections in the United States, 1979-1980. J Infect Dis 1982; 146:322-327.

6. Buchwald DS, Blaser MU: A review of human salmonellosis: II. Duration of excretion following infection with nontyphi Salmonella. Rev Infect Dis 1984; 6:345-356.

7. Haley CE, Guerrant RL: Institutional salmonellosis. Asepsis 1982; 4:7-12.

8. Chattopadhyay B, Pilfold JN: The effect of prolonged incubation of selenite F broth on the rate of isolation of Salmonella from feces. Med Lab Sci 1976; 33:191-194.

9. McCall CE, Martin WT, Boring JR: Efficiency of cultures of rectal swabs and faecal specimens in detecting Salmonella carriers: Correlation with numbers of Salmonellas excreted. J Hyg (Lond) 1966; 64:261-269.

10. Musher DM, Rubenstein AD: Permanent carriers of nontyphosa salmonellae. Arch Intern Med 1973; 132:869-872.

11. Nye FJ: Do antibiotics really prolong salmonella excretion? J Antimicrob Chemother 1981; 7;215-216.

12. Aserkoff B, Bennett JV: Effect of antibiotic therapy in acute salmonellosis in the fecal excretion of salmonellae. $N$ Engl J Med 1969; 281:636-640.

13. Joint Project by members of the association for the study of infectious disease. Effect of neomycin in non-invasive Salmonella infections of the gastrointestinal tract. Lancet 1970; 2:1159-1161.

14. Holmberg SD, Osterholm MT, Senger KA, et al: Drug-resistant salmonella from animals fed antimicrobials. $N$ Engl J Med 1984; 311:617-622.

15. Riley LW, Cohen ML, Seals JE, et al: Importance of host factors in human salmonellosis caused by multiresistant strains of Salmonella. $J$ Infect Dis 1984; 149:878-883.

16. Clementi KJ: Trimethoprim-sulfamethoxazole in the treatment of carriers of Salmonella. J Infect Dis 1973; 128(Suppl):S738-S742.

17. A comparison of the combination Pivmecillinam/pivampicillin and co-trimoxazole in the treatment of convalescent carriers of Salmonella and Shigella. Scand J Infect Dis 1984; 16:99-102.

18. Jick $H$ : Adverse reactions to Trimethoprim-sulfamethoxazole in hospitalized patients. Rev Infect Dis 1982; 4:4265-28.

19. Cohen ML, Fontaine RE, Pollard RA, et al: An assessment of patient-related economic costs in an outbreak of salmonellosis. $N$ EnglJ Med 1980; 299:459-460.

20. Levy BS: The economic impact of a food-borne salmonellosis outbreak. JAMA 1974; 230:1281-1282. 